\title{
Finite element method to solve engineering problems using ansys
}

\author{
Adrian Bogdan Șimon-Marinică ${ }^{1}$, Nicolae-Ioan Vlasin ${ }^{l}$, Florin Manea ${ }^{l}$, Gheorghe-Daniel \\ Florea $^{l}$ \\ ${ }^{1}$ National Institute for Research and Development in Mine Safety and Protection to Explosion - \\ INSEMEX, Petrosani, Romania.
}

\begin{abstract}
The Finite Element Analysis method, is a powerful computational technique for approximate solutions to a variety of real - world engineering problems having complex domains subjected to general boundary conditions. The method itself has become an essential step in the design or modelling of a physical phenomenon in various engineering disciplines. A physical phenomenon usually occurs in a continuum of matter (solid, liquid or gas) involving several field variables. The field variables vary from point to point, thus possessing an infinite number of solutions in the domain. The basis of finite volume method relies on the decomposition of the domain into a finite number of subdomains (elements) for which the systematic approximate solution is constructed by applying the variational or weighted residual methods. In effect, finite volume method reduces the problem to that of a finite number of unknowns by dividing the domain into elements and by expressing the unknown field variable in term of the assumed approximating functions within each element.
\end{abstract}

\section{Introduction}

Various phenomena treated in science and engineering are often described in terms of differential equations formulated by using their continuum mechanics models. Solving differential equations under various conditions such as boundary or initial conditions leads to the understanding of the phenomena and can predict the future of the phenomena. Exact solutions for differential equations, however, are generally difficult to obtain. Numerical methods are adopted to obtain approximate solutions for differential equations. Among these numerical methods, those which approximate continua with infinite degree of freedom by a discrete body with finite degree of freedom are called discrete analysis. Popular discrete analyses are the finite difference method, the method of weighted residuals, and the Rayleigh-Ritz method. Via these methods of discrete analysis, differential equations are reduced to simultaneous linear algebraic equations and thus can be solved numerically. 


\section{Basic concepts}

Ansys [1] is a general-purpose finite-element modeling package for numerically solving a wide variety of mechanical problems. These problems include static / dynamic, structural analysis (both linear and nonlinear), heat transfer, and fluid problems, as well as acoustic and electromagnetic problems. In general, a finite element solution may be broken into three stages and representative common engineering problems and their corresponding FEA discretization are illustrated in the figure below, fig. 1.

- Preprocessing: defining the problem: the major steps in preprocessing are to define the key points/lines/areas/volumes, define element type and material/geometric properties, as well as mesh lines/areas/volumes that are required. The amount of detail required will depend on the dimensionality of the analysis, 1D, 2D, axisymmetric, and 3D.

- Solution: assigning loads, constraints, and solving. Here, it is necessary to specify the loads (point or pressure), constraints (translational and rotational), and finally solve the resulting set of equations.

- Postprocessing: further processing and viewing of the results. In this stage we can obtain lists of nodal displacements, element forces and moments, deflection plots, and stress contour diagrams or temperature maps.
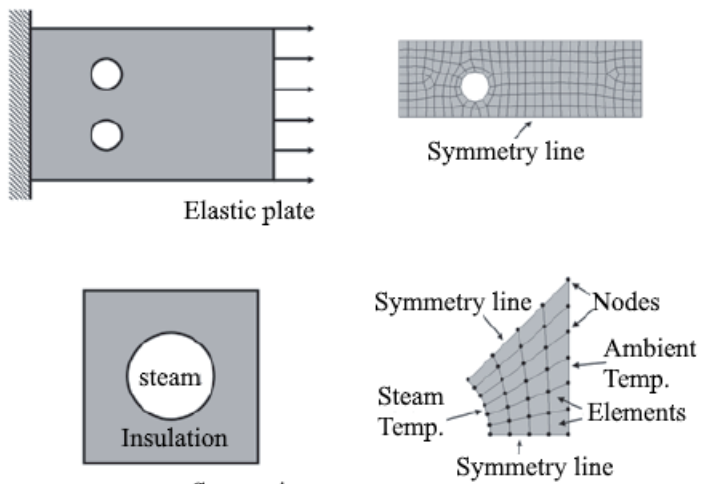

Steam pipe

Symmetry line

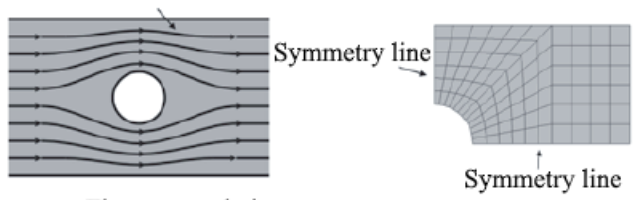

Flow arround pipe

Fig. 1. FEA representation of practical engineering problems.

There are three main approaches to constructing an approximate solution based on the concept of FEA: 
- Direct Approach. This approach is used for relatively simple problems, and it usually serves as a means to explain the concept of FEA and its important steps.

- Weighted Residuals. This is a versatile method, allowing the application of FEA to problems whose functionals cannot be constructed. This approach directly utilizes the governing differential equations, such as those of heat transfer and fluid mechanics.

- Variational Approach. This approach relies on the calculus of variations, which involves extremizing a functional. This functional corresponds to the potential energy in structural mechanics.
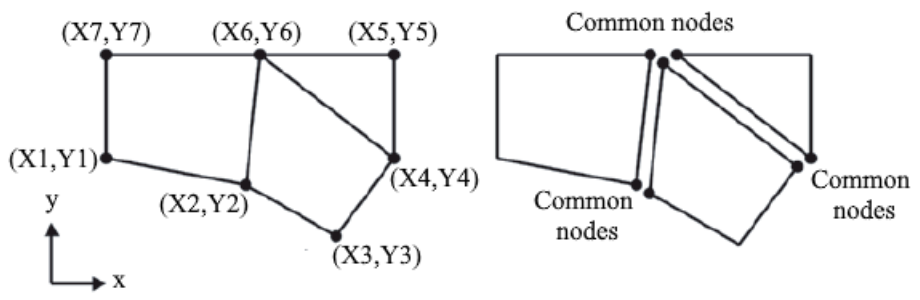

Fig. 2. Subdomains division of a domain.

\subsection{Common nodes}

The transformation of the practical engineering problem to a mathematical representation is achieved by discretizing the domain of interest into elements or known as subdomains, as shown in fig. 2. These elements are connected to each other by their common nodes. A node specifies the coordinate location in space where degrees of freedom and actions of the physical problem exist. The nodal unknown in the matrix system of equations represents one (or more) of the primary field variables. Nodal variables assigned to an element are called the degrees of freedom of the element. The common nodes shown in the figure above provide continuity for the nodal variables. Degrees of freedom of a node are dictated by the physical nature of the problem and the element type. In the following table 1 we find the degrees of freedom and the corresponding forces used in the finite element method for different physical problems.

\subsection{Common elements}

Depending on the geometry and the physical nature of the problem, the domain of interest can be discretized by employing line, area, or volume elements. Some of the common elements in FEA are shown in fig. 3. Each element, identified by an element number, is defined by a specific sequence of global node numbers. The specific sequence (usually counter clockwise) is based on the node numbering at the element level. The node numbering sequence for the elements presented in fig. 4 are presented in table 1 .

Table 1. Different engineering disciplines with degrees of freedom and force vectors. 


\begin{tabular}{|c|c|c|}
\hline Discipline & DOF & Force vector \\
\hline Structural/solids & Displacement & Mechanical forces \\
\hline Heat conduction & Temperature & Heat flux \\
\hline Acoustic fluid & Displacement potential & Particle velocity \\
\hline Potential flow & Pressure & Particle velocity \\
\hline General flows & Velocity & Fluxe`s \\
\hline Electrostatics density & Electric potential & Charge density \\
\hline Magnetostatics & Magnetic potential & Magnetic intensity \\
\hline
\end{tabular}

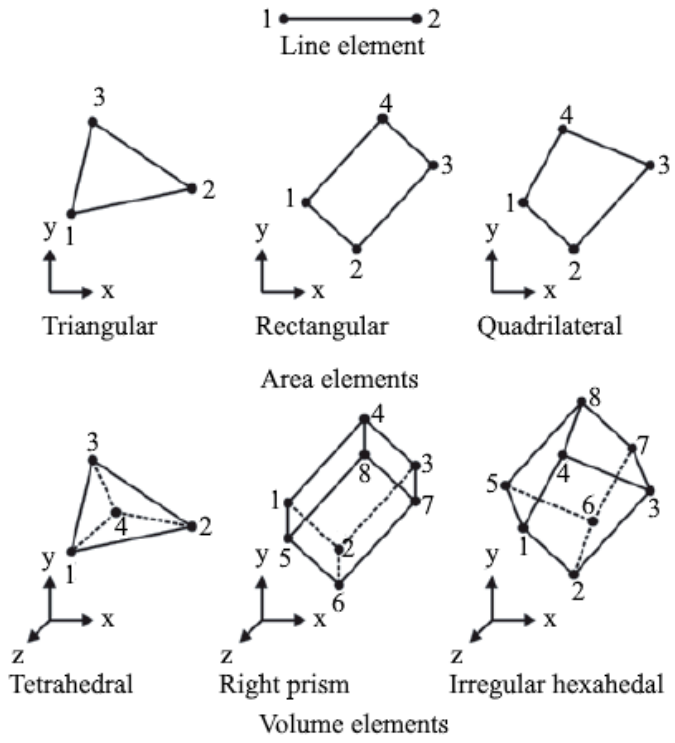

Fig. 3. Representations of elements with node numbers at the element level.

\subsection{Direct approach}

Although the direct approach is suitable for simple problems, it involves each fundamental step of a typical finite element analysis. Therefore, this approach is demonstrated by considering a linear spring system and heat flow in a one-dimensional (1 D) domain.
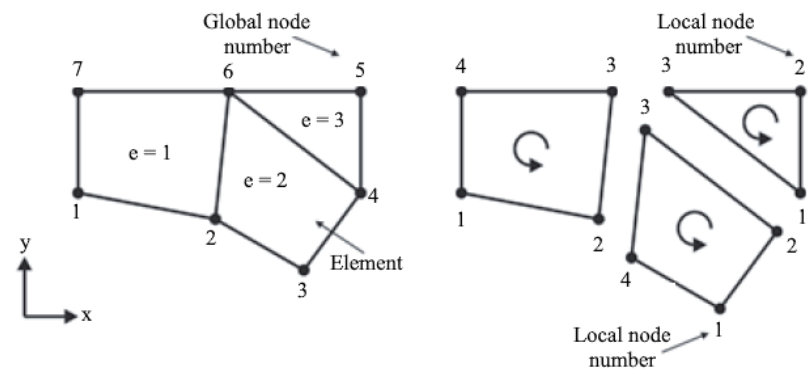

Fig. 4. Domain discretization with element and node numbering. 


\section{Practical Modeling Considerations}

The Ansys program is capable of simulating problems in a wide range of engineering disciplines. In order to reduce computational time [2], minor details that do not influence the results should not be included in the FE model. Minor details can also be ignored in order to render the geometry symmetric, which leads to a reduced FE model. However, in certain structures, small details such as fillets or holes may be the areas of maximum stress, which might prove to be extremely important in the analysis and design. Engineering judgment is essential to balance the possible gain in computational cost against the loss of accuracy. An example of practical engineering problem [3] with results from the computer simulation comes from the combustible gas diffusion case. In order to investigate the mode of diffusion and dispersion of the gas used in the experimental modeling of the formation, escape and ignition of the explosive mixture, a virtual geometry was made fig. 5, approximately identical to the room in which the physical experiment was performed. In order to avoid obtaining erroneous results [4], which lead to subsequent inadequate decisions, it is necessary to calibrate the mathematical models based on the physical experiments performed. The calibration process of the computer simulations [5], carried out using the Ansys FLUENT analysis package, starts with the input data, which must comply with the state conditions from which the physical experiment considered as reference starts.

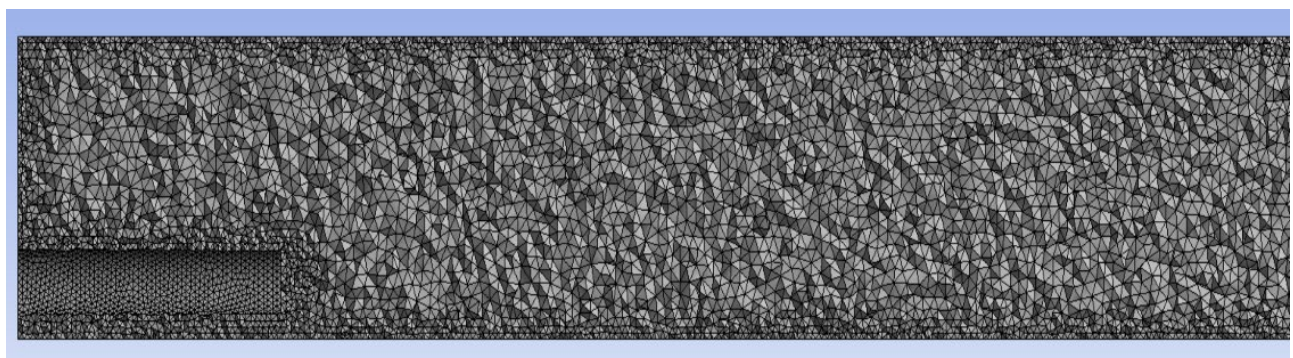

Fig. 5. High discretization of geometry used in simulation of combustible gas diffusion.

The virtual geometry consists of a rectangular chamber with small dimensions in order to obtain fast results. Inside the geometry we can observe a cylindrical body fig. 6 with a diameter of 10 millimeters, which was defined as an input parameter necessary for the gas flow inside the geometry. Regarding the simulation's objectives fig. 7 and fig. 8 a combustible gas leak inside a room, the case was defined as transient, density-based and with gravitational acceleration activated. The entire domain was set at a temperature of 15 degrees, a pressure of 101325 Pascals.

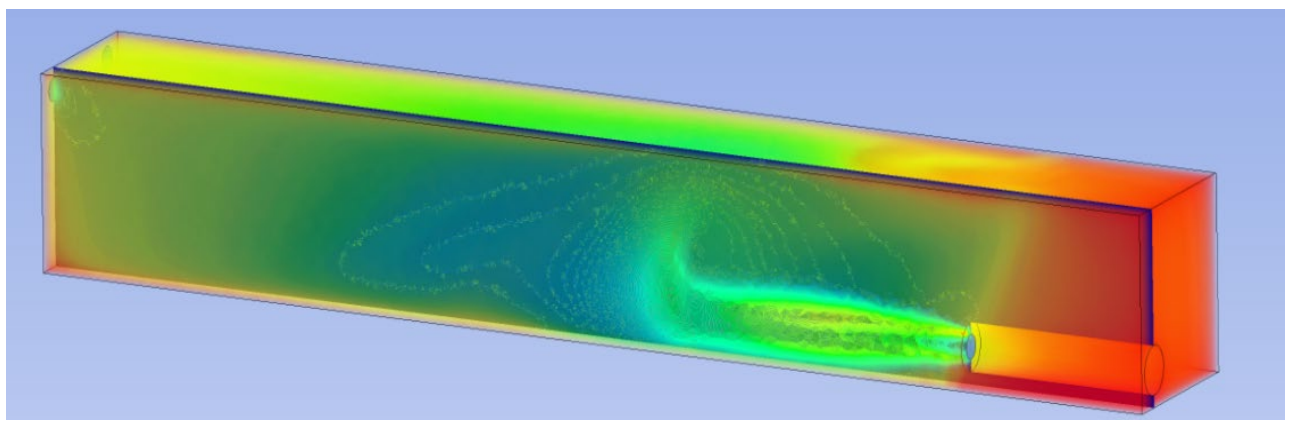

Fig. 6. Density contours of combustible gas at $t=4$ s inside the chamber. 


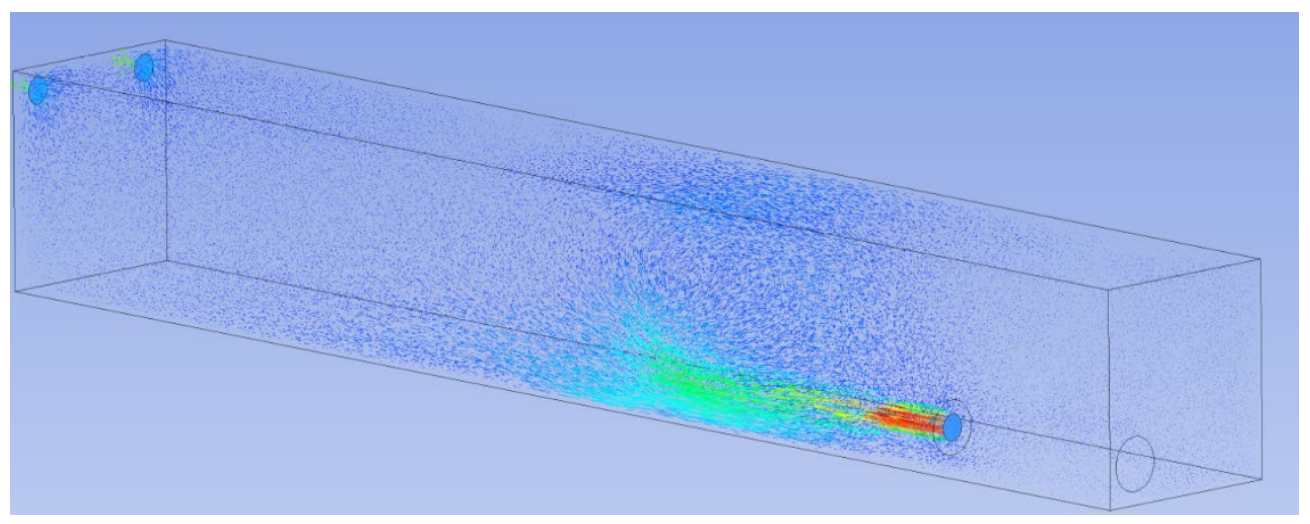

Fig. 7. Velocity vectors distribution.

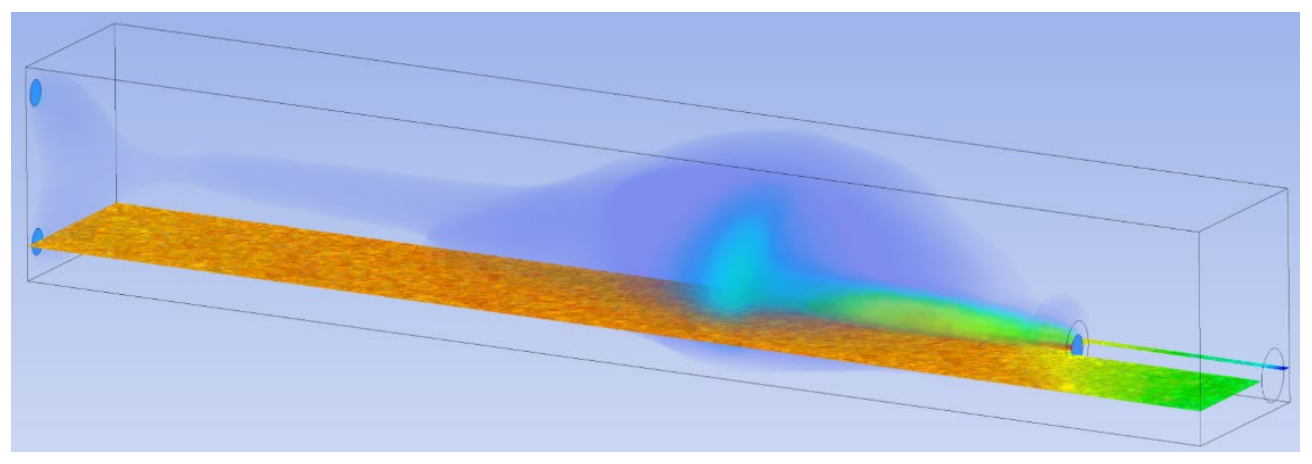

Fig. 8. Volume rendering of combustible gas leakage.

\section{Conclusions}

The parts we design work in a variety of real-working conditions, as under pressure, high temperature, under force, vibrations. We declare these conditions as boundary conditions in finite element method. Because of that we must define boundary conditions correctly to get correct results from our analysis. Some advantages of the finite element method are defined, such as the possibility to test our system for working condition with design data, before production and we can see the results, another advantage is that we can do optimization on design data for creating better product before production according to the analysis results and we can do a lot of different working condition tests on our product before production. The method of analysis is used in vast areas of industry such as space and aviation industry, automotive industry, bio medical, constructions. The Finite Element Method is a real engineering method and requires a good planning, a careful modelling, a correct approach that should be established for boundary conditions and other definitions, and the results should be evaluated and interpreted correctly. A Finite Element Analysis consists of the following steps: determination of analysis type, determination of material properties, preparing the numerical model of the design model, defining of loads and boundary conditions, evaluation and optimization. 


\section{References}

1. Ansys Software User Guide. Available on http://www.ansys.com/support

2. N.I. Vlasin, Simulări computerizate ale Exploziilor de Metan (INSEMEX, Petroșani, 2020)

3. A. B. Șimon-Marinică, E. Ghicioi, N. Vlasin, M. C. Șuvar, D. Florea, Proceedings of the 19th International Multidisciplinary Scientific Geoconference SGEM 2019 19, 1165-1171 (2019)

4. A. B. Șimon-Marinică, Z. Vass, Proceedings of the 9th Edition of the International Symposium on Occupational Health and Safety - SESAM 19, Petroșani, Romania (2019)

5. M. C. Șuvar, A. B. Șimon-Marinică, N. S. Șuvar, E. Ghicioi, A. Nicola, Proceedings of the 19th International Multidisciplinary Scientific Geoconference SGEM 2019 19, 471-478 (2019) 